\section{A SUMMARY}

or

\section{S U R G I C A L O P E R A T I O N S PERFORMED IN THE BRISTOL ROYAL INFIRMARY.*}

\section{By AUGUSTIN PRICHARD, EsQ.,} Surgeon to the Infirmary.

A FEW years ago, I published a series of reports of 875 operations, which I had performed up to the end of 1860, giving an account of hospital and private cases promiscuously; and then, among other points, I expressed my disbelief in the practical value of surgical statistics, in consequence of the difference of the cases. It is satisfactory to be able to say that in a large number of cases the success has been very great; but it proves of little or no aid and support to any other practitioner, when puzzled or hampered by the difficulties of a critical case. With the kind consent of my colleagues, I intend shortly to publish the actual results of the operations for the last eight years, and this will include those performed by all the surgeons at the Infirmary, but no private cases : the entire number is I I I5; and of these, six per cent. died.

The attention of the public has been directed lately to the question of hospitals, with reference to their construction, and management, and uses; and, without sufficient reason, a slur has been cast on the large and old hospitals, which some even of our own profession assert do more evil than good, or, at any rate, destroy many lives which would otherwise be saved; and, according to the fashion of the time, a new word has been coined, namely, hospitalism, the meaning of which I do not know, except that it expresses something about hospitals in rather a contemptuous tone.

I think, however, that until England increases in area or lessens in population, and while men are collected together in cities and towns, or as long as railways and buildings exist, and while ships, and commerce, and engineering achievements of any kind, require men to work them, and while disease and acts of violence occur from human vice, so long will the existence of large hospitals in our towns be a necessary part of our civilised life. The time for their disestablishment is still far away.

And more than this; although I am about to tread on very delicate ground, I must be allowed to express my conviction that the very great experience afforded by a large hospital does more for the advance of the science of medicine and surgery, and their successful practice, by being concentrated in a comparatively small number of men, than if it were equally diffused among the whole body, and that the whole profession, as well as the public, are benefited by this arrangement. If, for example, each of us had to perform, during his lifetime, as his share, one amputation, two or three cataract operations, one lithotomy, and one hernia operation, how could the varied experience be brought together so as to deduce new and better modes of performing these operations? By the records and experience of private practice alone, how could Lawrence have written his exhaustive book on Hernia, where you may find an account of every known form of the disease; or even in Diseases of the Eye, a so-called speciality, the largest private practice would not enable the surgeon to classify and describe thoroughly the disorders to which the eye is liable.

In these remarks I have naturally taken my examples from the surgical side of the question; but they equally apply to the internal diseases which are usually under the care of the physicians. For instance, obscure cerebral, or pulmonary, or renal disorders, treated scientifically and with equal success, as isolated cases or in small numbers in private practice, give little or no opportunity for general deductions as to the comparative value of different modes of treatment, and still less scope for the study of morbid appearances or the scientific part of the subject.

It would be absurd in a meeting like this to speak of the necessity of large hospitals as means of study. I know that we have sent out from our Bristol school in my time a large number of men qualified and willing to undertake all the most urgent and important duties of medical and surgical practice, who are largely occupied in their work. On the other hand, I maintain that it is the paramount duty of those concerned to publish unreservedly, in the proper channels, the medical journals, but not in the public press, all new points of scientific and practical interest as they become developed and are confirmed by hospital experience.

\footnotetext{
* Read before the Bath and Bristol Branch.
}

I must add one word about the alleged evils of old hospitals, and their poisonous walls and atmospheres. We claim for our Bristol Royal Infirmary that it is one of the oldest, if not the oldest, hospital established in the provinces, and that it is one of the largest. I believe that the death of a patient, because of his presence in the wards, is an event of extreme rarity. We get a case of pyæmia now and then, and our patients recover not unfrequently from it; but I do not attribute the disease to the place, but to the injury and the patient's state of health. I have seen several fatal cases of pyæmia in private practice in the freshest country air.

And then, as to the success of treatment generally, I find by last year's report of our Infirmary, that we had 1434 in-patients under treatment in the surgical wards, among which were cases of compound fractured legs and arms, lacerated limbs, and other machinery accidents ; burns, fractured skulls, cancerous cases, operations for stone, eight operations for strangulated hernia, half-a-dozen thigh-amputations, and tumours, and wounds, and surgical diseases of all kinds, and the entire mortality was 37 , or 2.6 per cent. ; and I therefore conclude that the size and age of a hospital have not the deleterious influence assigned to them, and that our patients do not die of hospitalism.

It would be tedious and unprofitable to read to you tables of names and figures referring to eight years of operations, which 1 have prepared; but I think it might be of interest to take some of the most im. portant operations and their results, and I have selected those which comprise the great majority of deaths.

Out of 55 cases of Epithelioma of the Lip operated on, 2 died. This operation is generally considered almost devoid of danger; but among this large number were several where the disease had extended along the whole or the greater part of the lip, and operations to restore the lip were necessary; in two of them the disease was in the upper lip, and one of the patients was 8I years of age. This disease is generally very easily diagnosed, is practically incurable by applications, but readily and safely removable by the knife. When once the glands under the jaw have become large and hard, although you may remove the primary disease, your patient will inevitably die a frightful and painful death from cancer of the face and neck. If the disease be removed freely and early, the cure is often permanent. I therefore consider that surgeon inexcusable who does not urge on his patients the imperative necessity of an operation without wasting precious time in what he ought to know to be hopeless treatment.

Our Hernia cases are the most fatal series of diseases which we have to treat in hospital practice, and in these private patients certainly have the advantage; not because of hospital air, but because of the loss of strength and of invaluable time which the patient suffers, by a painful removal, often from a considerable distance, in a state in which strength and time are life itself. Out of 19 cases of inguinal hernia, I I died; and out of 21 cases of femoral hernia, 6 died; and of that rare and generally fatal form, strangulated umbilical hernia, we had two cases, and both recovered: altogether, in decimal language, 60 per cent. recovered. We have operated, during these eight years, on 30 cases of reducible hernia, for the radical cure. Most of the cases have been in children, and the great majority have done well. There have been no alarming symptoms. We perform the old operation generally; viz., Wuitzer's, and where it has not succeeded in curing the hernia, it has relieved the patient so as to render a truss efficient, and thus give a promise of ultimate cure.

We have had 43 operations for the Removal of Tumours of the Breast; 23 were for scirrhus, the rest for non-malignant tumours-chronic mammary, hydatid, and cystosarcoma. One died out of the whole number. On the most important point, as to the return or otherwise of the disease, I am sorry to say that I can throw no light.

We have had Io of those dreadful cases of Extravasation of Urine from rupture of the urethra and perineal sloughing, with a discharge of putrid urinous pus, where it was necessary to open the perinæum and scrotum. Six of these were fatal; and, by a coincidence, five of them fell to the lot of one surgeon.

There have been as many as 9 cases of Amputation of the Penis for cancerous disease. All did well; but I am not in a position to tell the ultimate result, which is a most important point. Some, I know, are well now. By a similar coincidence, the great majority of these cases came under the hands of one surgeon, who thus has had an unusual chance of attaining skill and eminence in the performance of this disagreeable operation.

In the Operations on the Eyes, which were not very numerous, we had of course no deaths. There were 34 cases of removal of the globe of the eye to preserve its fellow; and, as the operation is one in which I take special interest, it is a pleasure to add that they were all successful. In cancerous cases, whether they be melanosis or encephaloma, an operation is only palliative if the disease has broken through 
the coats of the eye and spread into the tissues of the orbit. When the disease is confined within the sclerotic, I believe that an operation may be a permanent cure.

We had 66 operations for the Removal of Necrosed Bone: one of the patients died. Of the whole number, no fewer than 23 were cases of disease of the tibia.

Of seven instances where we operated for the cure of Ununited Fracture of the limbs, two were of the humerus, two of the femur, two of the forearm, and one of the leg. One of the patients died. The plan which we usually adopt is to turn out the ends of the bone in the most convenient direction, and cut them off; and, after drilling holes through them, to tie them together with wire, or, what is better, twisted horsehair, like a fishing-line, and bring and keep the parts in apposition. The bone that we have to remove is generally smooth on the surface, and has a very thin compact layer outside, with cancellous structure inside. It is remarkable how well these ununited fractures of the forearm do, when we consider the amount of damage which the softer parts must suffer whilst the four ends of the fractured bones are being exposed and perforated for the ligature. I always look upon the forearm as a part which bears accidents and operations better than most others.

We had 4 deaths from Compound Fracture of the Skull, in which portions of loose bone were removed, without relief of the symptoms. One of these was trephined.

There were I 5 cases of Excision; namely, 8 of the knee and 7 of the elbow. There were no deaths; but two or three of the knee-cases required amputation subsequently. There were several patients who recovered, and are well now, with strong legs; but I regret that I cannot give the exact number. The cases of excision of the elbow were still more successful-that is, they all recovered. In two instances which I saw some years afterwards, there was too little union at the new joint, rendering artificial support at the elbow necessary for work.

The cases of Ligature of Arteries comprised six instances of ligature of the femoral artery, all successful. Five were performed for aneurism, and one for elephantiasis, which was much relieved. There were two cases of ligature of the external iliac artery for femoral aneurism, both successful ; and one instance of traumatic aneurism of the ulnar artery, requiring an operation for its cure. The subject of one of the iliac artery operations was also one of the five who had the femoral tied, for the disease appeared in one limb after it had been cured in the other.

The only unsuccessful case was one in which I tied the common carotid for repeatedly recurring hæmorrhage from a stabbed wound in the neck. The patient died, worn out by loss of blood cuming on from a point deep in the neck, at intervals of two, or three, or more days. It appeared, on dissection, that the point of the assailant's knife had passed in between the atlas and the occipital bone, severing the vertebral artery completely, in the middle of the fibrous tissue which binds these bones together. I question whether a similar case ever occurred ; but if it could be diagnosed, no ligature could be applied which would touch the evil. The treatment would be such a lateral position of the neck as to press the bleeding vessel between the two bones. In my case, now and then as much as ten days passed without any bleeding : and I believe that the patient's head pressed on the artery during that time, and restrained it.

The operations for the removal of Tumours were 94 in number, being exclusive of tumours in particular organs, such as the breast or testis ; and the number of deaths was five, two of them from cancerous disease, and one after the removal of a steatomatous tumour of the scalp. This patient was attacked with erysipelas of the scalp some days after the operation, and died; and this was the only instance where there could be any possible suspicion that hospital influences hastened his death. The number of operations for the class of tumours which come, with steatoma, under the head cystoma, was 25 .

We had two cases of Cysticercus Cellulosæ, one being in the cheek and the other in the thumb. There is a peculiar hardness, and mobility, and elasticity, in these little tumours, which enable us frequently to diagnose them before operation; and thus we can make a careful incision if we want to see a perfect specimen.

One of the fatal results was seen in a man who had an enormous Fibrous Tumour on the anterior or ventral surface of the scapula, projecting into the axillæ. A formidable operation was required for its removal, and the patient sank.

Lastly, I come to the surgeon's last resource, Amputation ; and I have to report that in the eight years, exclusive of single fingers and toes, but inclusive of all amputations of parts of the hands, or of the foot, as Chopart's operation, of the forearm, arm, shoulder-joint, ankle, leg, and thigh-in fact, all the principal amputations-12O, of which 15 died.

Amputations of the forearm, leg, and ankle, with us are very little fatal. In 9 amputations of the forearm, 4 were primary-that is, per- formed immediately for accidents, and none died; out of 18 at the ankle-joint, 7 being primary, $I$ of the latter died.

Of 23 cases of leg-amputations, two died. One of the patients was a man who had both legs crushed, requiring immediate operation, and he had fractured skull as well; the other operation was done for gangrena senilis, offering little or no chance from the first. Of the 21 successful cases, one was a man aged 27 , who had both legs amputated at the same time for compound comminuted fracture. I have counted him as one successful case, although perhaps he ought to count as two; but then I thought that some objectors might say that the patient who died with fractured skull, after double amputation, ought to reckon as two deaths.

My statement, therefore, that these operations are not very fatal, rests on this fact, that in $\mathbf{5 2}$ cases there were 3 deaths, 2 of which had nothing to do with the operation, as it was inevitable under any circumstances. In my own practice, I have never yet lost a case of amputation of the forearm or ankle-joint.

Of 13 arm-amputations, 6 were primary, and 7 were for chronic disease, and one of each died. The fatal cases were a severe burn up to the shoulder, and a cancerous case.

There was one amputation at the shoulder-joint, which did very well.

The amputations of the thigh were 47 , and the deaths 8 , two of them being strumous disease in very weakly subjects, where the joint had been laid open, with the hope of getting some healthy action. Three of the other fatal cases were primary amputations.

The amputation at the knee-joint seems to promise well. Two are included among these thigh-operations. Let me warn any whom it may concern to be very tender with the necessarily thin and long anterior flap, and to avoid pressure on it, and to leave plenty of posterior flap as well.

Among our cases, there have been all varieties of operation-flap, circular, and Teale's rectangular. We have used ligature, torsion, and acupressure, to stop the bleeding; and carbolic acid, chloride of zinc, and alcohol, to check suppuration; and I cannot say that any one plan is decidedly better than the others. For myself, I have seen no reason to change my old predilection for the flap-amputation, cutting from without inwards, and then transfixing the limb, and leaving a long anterior flap; and I still use the weak chloride of zinc solution over the surface of the flaps. In stopping the blood, I never knew a properly applied ordinary well-waxed ligature do any harm. Horse-hairs are the best sutures. I have not much used the carbolic acid lotion and putty system, but some of my colleagues have, and report favourably of it. The weak carbolic acid lotion is invaluable for syringing into chronic abscesses, and for enveloping a limb with saturated wool, immediately the pus has been let out.

I have thus given a somewhat hurried account of our principal operations, and the list comprises nearly all the fatal cases. The whole number was I I I 5, and deaths 68 , being 8 and a fraction each year.

I am afraid that my paper has been rather numerical ; but the numbers have been given to show what work we have actually done, rather than with a view to statistics, my distrust of which I expressed in the beginning; and I consider that I have sufficiently shown my disrespect for them by bringing under the head of operation cases, and including among the 68 deaths, a considerable number of hopeless surgical maladies which underwent some treatment in our operation-room, and were, therefore, entered in our operation-book: thus I have included some tracheotomy cases performed for diseases almost inevitably fatal, as diphtheria and aneurism, 6 cases of extravasation of urine, 4 cases of fractured skull, and 2 of dislocated hip, in one of which the patient died of aneurism of the aorta.

Among other causes, I attribute these good results to the lightness and cleanness of our wards, and to the excellent ventilation of the house, which is as perfect as possible in a building containing between 300 and 400 inhabitants, in the middle of a densely populated district of the town. The corridors on each floor, which run the whole length of the building in a direct line, are open at the east and west ends by large windows, and the windows of the wards are at right angles to these, and therefore north and south, and opposite to each other. Thus we can have the air admitted from whatever quarter it blows. This plan of ventilation is inartificial and primitive; but it has the great advantages of simplicity and efficiency.

I must, before I conclude, bear my witness that men who lead temperate lives, or even lives of total abstinence from fermented liquors, make speedier recoveries and bear accidents and operations better than those who drink freely; and no doubt excessive drink has been a help towards a fatal result in some of our cases. I also think that at many of the factories in Bristol, and no doubt elsewhere, breweries, sugar-refineries, and other places, an unnecessarily large allowance of beer is made to the men, and great evil is caused by it. At the same time, we 
cannot satisfactorily treat severe surgical cases without the help of alcohol.

Numerous improvements in this age of active change are being made in the construction of our hospitals, and alterations in the system of nursing, which will lead hereafter to greater success in treatment, and a somewhat diminished death-rate, for that is the object we have in view; and I am rejoiced to think that it is so; but, so long as men get their bodies and limbs crushed and require amputations for compound fractures, or get their intestines pinched by a hernial stricture until the vitality of the part is hopelessly damaged, so long must there be deaths after operations; and, to repeat what I said at the beginning of my paper, I do not believe that large hospitals can be done away with, nor that, even when they are old as well as large, they deserve altogether the bad name that has been given to them.

\section{THE INFLUENCE OF MCDERN DOCTRINES OF INFLAMMATION UPON ITS TREATMENT.*}

\section{By WILLOUGHBY FRANCIS WADE, M.B., B.A.,} Physician to the General Hospital, Birmingham.

ABSTRACT questions of pathology are perhaps more suited for quiet reflection at home, than for discussion at a meeting of this kind. At the same time we can scarcely fail to derive some advantage from the consideration of the doctrines of a process which, even now that it has been shorn of some of its vast proportions, still dominates over a large region of both medicine and surgery. The light which the vigorous nineteenth century questioning of nature has shed upon many regions of pathology, has illuminated none more highly than this. Morbid anatomy has shown that inflammation may exist, without giving rise to those symptoms by which its existence is, during life, best attested for the purposes of treatment.

The microscope has contributed in a high degree to more accurate knowledge of the minutiæ of the inflammatory process. Indeed, we may go much further, and say that, without this instrument, these minutiæ would be unknown and unknowable.

Observation of the natural course and progress of inflammations, in cases where they have been undisturbed by the administration of drugs, has furnished data of the utmost value to practitioners. Lastly, theories of inflammation, more especially those which involve a consideration of its relation to normal nutrition, have indisputably produced an effect upon the practice of us all, and in that of some of us have induced modifications so considerable, that it becomes of the last importance to inquire how far they are justified by clinical facts, and how far, also, they are logically justifiable, i.e., how far they are necessary conclusions flowing from these theories.

The relation between fact, theory, and hypothesis, is well determined and simple; but it is so constantly overlooked in discussions of this kind, that I may be pardoned for briefly referring to it. Theory does not seek to make or invent facts, but only to arrange them, to determine their order, sequence, and etiological relation-how far any of them stand to others in the light of cause and effect. Facts without this arrangement, i.e., without theory, are disorderly, chaotic, and of limited applicability.

Hypothesis, on the other hand, does invent (if we may use such an expression) facts, or rather it imagines that there may be facts, the existence of which is unproven. It does this to support a theory for which a sufficient number of ascertained facts do not exist.

Without staying to discuss the merits and demerits of hypotheses, and they have both, it is sufficient to remark that the principal danger of them is, that we too often fail to remember that they embody fictitious, and not real, facts. We may, with but little alteration, say of them, as has been said of words, they "are not things, but are very apt to become so."

The principal theorists whose views have, of late years, had a decided influence upon our practice, are Bennett and Virchow. One great object of both these able pathologists has been to determine the starting point of inflammation, and another to determine its essence.

Seeing that inflammation is a process-which word indicates that it may be divided into several steps, stages, or parts-and seeing that this process is one of great complexity, it may be doubted, and I for one do doubt, that any of these steps, stages, or elements (not even excepting the migration of the white corpuscles), will ever be found, which is of such pre-eminent importance as to entitle it to this latter distinction.

Still, in a less extended sense, there may be, and probably is, such

* Read before the Birmingham and Midland Counties Branch. an essential element; in this way-all admit that, during the course of an inflammation, there are certain conditions of the local blood-vessels, which are also found in forms of disease to which the term inflammation has never been applied or considered applicable. In what way, e.g., does inflammation differ from congestion or determination of blood? To this question, Dr. Bennett would answer-by the presence of exudation. And hence exudation might be termed the essentiai element of inflammation, as contrasted with congestion. This is a limited use of the term; whereas he considers exudation to be the essential element of inflammation, without any limitation. But here arises a difficulty : Bennett himself points out that in other cases there is an exudation from the vessels, as in cancer and tubercle, from which it follows that, admitting exudation to be the essential element of inflammation, we have between it and tubercle, or cancer, no line of demarcation, except the different subsequent developments of the exudation. We find also simple exudation, i.e., exudation of the same characters, and with the same developmental tendencies, as the inflammatory, occurring in so orderly a manner, and with such an absence of disturbance of the circulation or of the nervous system, that no one would call it inflammation, and it could scarcely, if at all, be distinguished from ordinary nutrition. We come, then, to this : either everything is inflammation, or nothing. Dr. Bennett is disposed to choose the latter alternative, and to dispense with the term and idea of inflammation altogether.

I think these facts of great importance in a practical point of view, but would draw other lessons from them than those taught by Bennett. Here, I think, is a flagrant instance of words becoming things. It would be highly satisfactory to abolish inflammation by the act of a pathological parliament, if we could thereby abolish pneumonia, and peritonitis, and whitlow. As we cannot do so, we must see what more practical conclusions we can draw.

In the first place, we may see most plainly that there are all grades of the exudatory process, some of which so closely approximate to normal nutrition that they are undiscoverable by ordinary means, and demand no treatment. On the other hand, there are grades so tumultuous and disorderly in their development, that they are attended by phenomena, which render their recognition facile, and cry out for removal and relief. We must, then, as practitioners, and, I am bold to say, as pathologists, recognise two classes of inflammation, which we may, till some more precise phraseology be invented, rudely term : Ist. The pathological class; 2nd. The practical class. Of this latter, the prominent features are still, as in the days of Celsus, pain, heat, redness, and swelling. In the next place, we find that between these two classes there is no positive line of demarcation. Further, we must admit that, between the former of these classes and healthy orderly nutrition, there is no positive line of demarcation.

The absence of any abrupt line of separation may at first sight seem to the practitioner regrettable. It is not so; on the contrary, those who have practised longest, best know the limited range and powers of the weapons with which we fight. This continuity of actions encourages us to feel and remember the great restorative powers of nature, nowhere more visible than in the case of this pathologue of which we speak. Nature can often, unassisted, reduce to order the impetuous disorder of the inflammatory current; the more certainly so if we can, as we often may, remove the cause which first diverted nutrition from its normal course, or, if we can, as we often may, break one link of the chain of disordered action. We thus find that, although these theorefical views, pushed to undue and extravagant lengths, confound and paralyse us as practitioners, yet, on the contrary, when held soberly and moderately, they encourage and strengthen us.

It is not needful to enter at great length upon an exposition of the peculiar doctrine of Virchow. A few words will suffice. Exudation undergoes certain modifications and developments, such, for example, as its change into pus. Exudation is homogeneous and structureless; pus consists of cells floating in a liquid. Bennett believes that these cells arise from an aggregation of molecules spontaneously taking place in the exudation. When this latter, on the other hand, becomes converted into fibroid structure, he believes the fibres also are formed by molecular aggregation.

Virchow, on the contrary, holds that these structures-the pus-cell and the fibre-are offshoots from the original cells of the part, and that cell-growth may be the only microscopical phenomenon, as it is, according to him, the essential element of inflammation. This cell-growth is, however, tumultous and irregular, the formative process being unduly active, the developmental one deficient. Hence, Virchow's theory $i$ called the "cell-theory": his watchword being "Omnis cellula cellula"-every cell is born of a cell. It does not arise from any spon taneous aggregation of molecules, as Bennett would have us believe. $D$ have no intention of raising the question, which of these views is the 